\title{
Reduced cerebral blood flow and white matter hyperintensities predict poor sleep in heart failure
}

\author{
Michael L Alosco ${ }^{1}$, Adam M Brickman², Mary Beth Spitznagel', Erica Y Griffith², Atul Narkhede ${ }^{2}$, Ronald Cohen ${ }^{4}$, \\ Lawrence H Sweet $^{5}$, Joel Hughes ${ }^{1,3}$, Jim Rosneck ${ }^{3}$ and John Gunstad ${ }^{1 *}$
}

\begin{abstract}
Background: Poor sleep is common in heart failure (HF), though mechanisms of sleep difficulties are not well understood. Adverse brain changes among regions important for sleep have been demonstrated in patients with HF. Cerebral hypoperfusion, a correlate of sleep quality, is also prevalent in HF and a likely contributor to white matter hyperintensities (WMH). However, no study to date has examined the effects of cerebral blood flow, WMH, and brain volume on sleep quality in HF.

Methods: Fifty-three HF patients completed the Pittsburgh Sleep Quality Index and underwent brain magnetic resonance imaging to quantify brain and WMH volume. Transcranial Doppler ultrasonography assessed cerebral blood flow velocity of the middle cerebral artery (CBF-V of the MCA).

Results: $75.5 \%$ of HF patients reported impaired sleep. Regression analyses adjusting for medical and demographic factors showed decreased CBF-V of the MCA and greater WMH volume were associated with poor sleep quality. No such pattern emerged on total brain or regional volume indices.

Conclusions: Decreased cerebral perfusion and greater WMH may contribute to sleep difficulties in HF. Future studies are needed to confirm these findings and clarify the effects of cerebral blood flow and WMH on sleep in healthy and patient samples.
\end{abstract}

Keywords: Sleep quality, Heart failure, MRI, Brain perfusion, White matter hyperintensity

\section{Background}

Heart failure (HF) affects greater than 5 million American adults and is associated with poor outcomes such as cognitive impairment, decreased quality of life, rehospitalizations, and increased morbidity and mortality risk [1-3]. As many as $80 \%$ of HF patients also report experiencing sleep difficulties [4] that are multifaceted in nature, including difficulties falling asleep, insomnia, interrupted sleep at night (e.g., nocturia), restless sleep, and breathing difficulty during sleep [5-10]. Poor sleep quality in HF, in-turn, is associated with decreased quality of life, depression, impaired self-care, and decreased participation in key treatment recommendations (e.g., physical activity) $[11,12]$.

Much attention has been paid to correlates and modifiers of poor sleep quality in HF. Sleep quality in HF is affected by an array of demographic, medical, and clinical factors.

\footnotetext{
* Correspondence: jgunstad@kent.edu

'Department of Psychology, Kent State University, Kent, OH, USA

Full list of author information is available at the end of the article
}

Demographic variables that negatively influence sleep in HF include older age and being female $[5,7,13]$. In terms of medical factors, taking beta-blockers and medical comorbidities such as respiratory disease, stroke, hypertension, and coronary artery disease have been linked with sleep disturbances [5]. Many of these comorbidities are theorized to negatively affect circadian rhythms subsequent to associated disabilities and limited daytime activity [14]. Known psychosocial modifiers of sleep in HF include depression, anxiety, and perceived health status $[5,13]$. Despite these findings, the literature on factors that influence sleep quality in HF is not entirely consistent $[5,7,13]$, suggesting poor sleep quality in HF may be more complicated than typically believed.

Although not yet examined, a likely mechanism of poor sleep in HF may involve structural and functional brain abnormalities. Adverse brain changes such as brain atrophy, white matter hyperintensities (WMH), and cerebral hypoperfusion are common in HF and believed to underlie cognitive impairment in this population $[15,16]$. Interestingly, 
recent work also shows poor sleep is associated with reduced cognitive function in HF [11] and HF patients exhibit reduced structural (e.g., atrophy) and functional (e.g., impaired axonal projections) brain integrity among key brain regions that help regulate sleep (e.g., raphe magnus, hypothalamus, medial temporal lobe structures) [17-20]. Cerebral hypoperfusion is commonly proposed to underlie adverse brain changes in HF, including white matter hypertintensities, and may also impact sleep quality in $\mathrm{HF}$ through its impact on the brain and sleep regulation $[15,21]$. Indeed, altered cerebral hemodynamics, brain atrophy, and WMH have all been independently correlated with sleep difficulties and regulation (e.g., excessive daytime sleepiness, difficulties with breathing during sleep, arousals during sleep) in other populations [19,22-25].

Despite these findings, the mechanisms of sleep difficulties in HF patients remain poorly understood. The purpose of the current study was to examine the effects of cerebral blood flow, WMH, and total brain volume on sleep disturbances in older adults with HF. This study also examined the possible association between sleep quality and volumes of regional brain structures believed to be important for sleep (e.g., thalamus and brain stem) $[19,26]$. Based on past work in other samples, we hypothesized that adverse structural brain changes (e.g., greater WMH, smaller total and regional brain volume) would be associated with greater sleep disturbances in HF due to their known role in sleep regulation. We also hypothesized that reduced cerebral blood flow would predict poorer sleep quality in HF in light of its known association with brain insult (e.g., WMH) in this population.

\section{Methods}

\section{Participants}

A total of 77 patients with HF were recruited for a NIH funded study examined cardiac rehabilitation and neurocognitive outcomes in HF. All participants were recruited from primary care and cardiology practices at Summa Health System in Akron, Ohio, and reflect the HF population receiving treatment at that facility. Data collection for this study occurred between 2009 and 2012. Throughout the study, HF participants completed assessments over a one-year period. For this cross-sectional study, only baseline assessments among a subset of non-cardiac rehabilitation controls that underwent neuroimaging were examined.

The participant sample size of the current study was reduced to 53 due to missing data on study variables and on individual items of the Pittsburgh Sleep Quality Index (PSQI) used to calculate the global composite, and/or invalid responses on the PSQI. Invalid responses included $>100 \%$ in the calculation of component number 4 of the PSQI (i.e., percent of time spent sleeping while in bed). Excluded participants did not differ in age $(t(75)=-1.50, p=0.14)$, gender $\left(\chi^{2}(\mathrm{df}=1)=1.02, p=\right.$
$0.31)$, education $(t(75)=-1.19, p=0.24)$, cerebral blood flow velocity $(t(68)=0.01, p=0.99)$ or in terms of medical comorbid history, including hypertension $\left(\chi^{2}(\mathrm{df}=1)=\right.$ 3.84, $p=0.05)$, sleep apnea $\left(\chi^{2}(\mathrm{df}=1)=0.06, p=0.80\right)$, and diabetes $\left(\chi^{2}(\mathrm{df}=1)=2.32, p=0.13\right)$. However, excluded participants had a higher left ventricular ejection fraction $(t(74)=2.45), p=0.02 ; \mathrm{M}(\mathrm{SD})=49.43(14.34)$ vs. 40.98 (13.57)).

Strict inclusion/exclusion criteria were chosen for entry into the larger NIH funded study. Inclusion criteria were as follows: ages of 50-85 years, English as a primary language, and a diagnosis of New York Heart Association (NYHA) class II or III at the time of enrollment. Potential participants were excluded for any contraindications to magnetic resonance imaging (MRI) (e.g., pacemaker), history of head injury with more than 10 minutes loss of consciousness, Axis I psychiatric disorders (e.g. schizophrenia, bipolar disorder), substance abuse and/or dependence, and renal failure. Participants were also excluded for a history of significant neurological disorder (e.g., dementia) and the current sample exhibited an average Mini Mental Status Examination score of $27.72(\mathrm{SD}=1.96)$. Inclusion and exclusion criteria were determined through selfreport and corroborated and supplemented by a thorough medical record review. See Table 1 for demographic and medical information.

\section{Measures}

\section{Sleep quality}

The Pittsburgh Sleep Quality Index (PSQI) was used to assess sleep quality in the current sample [27]. The PSQI

Table 1 Demographic, medical, and clinical characteristics of $\mathbf{5 3}$ older adults with heart failure

\begin{tabular}{cc}
\hline \multicolumn{2}{c}{ Demographic characteristics } \\
\hline Age, mean (SD) & $69.81(8.79)$ \\
Sex (\% Women) & 37.7 \\
Race (\% Caucasian) ( $=51)$ & 86.8 \\
Education, mean years (SD) & $14.17(2.87)$ \\
\hline \multicolumn{1}{c}{ Medical and clinical characteristics } \\
\hline LVEF, mean (SD) & $70.98(13.57)$ \\
Sleep Medication (\%) & 24.5 \\
Diabetes (\%) & 66.0 \\
Hypertension (\%) & 26.4 \\
Sleep Apnea (\%) & 17.0 \\
Depression (\%) & $66.02(22.56)$ \\
2-minute step test, mean (SD) & $42.89(12.94)$ \\
CBF-V MCA, cm/s mean (SD) & $8.42(3.57)$ \\
Global PSQI, mean (SD) & \\
\hline
\end{tabular}

Note. LVEF left ventricular ejection fraction, CBF-V MCA cerebral blood flow velocity of the middle cerebral artery, PSQI Pittsburgh sleep quality index. 
is a 19-item self-report measure that generates 7 components of sleep quality, including subjective sleep quality, sleep latency, sleep duration, habitual sleep efficiency, sleep disturbances, use of sleeping medication, and daytime dysfunction. The sum of these components yields a global PSQI score that range from 0 to 21 . Higher scores reflect poorer sleep quality and a score $>5$ is a sensitive and specific predictor of impaired sleep [27]; this cut score was used to help characterize the sample and the continuous global PSQI served as the dependent variable. The PSQI demonstrates strong psychometric properties, including internal consistency, test-retest reliability, and is widely used in medical populations including $\operatorname{HF}[5,11]$.

\section{Neuroimaging}

Whole-brain, high-resolution 3D T1-weighted images (Magnetization Prepared Rapid Gradient-Echo, MPRAGE) were acquired on a Siemens Symphony 1.5 Tesla magnetic resonance imaging scanner for morphologic analysis. Twenty-six slices were acquired in the sagittal plane with a $230 \times 100 \mathrm{~mm}$ field of view. The acquisition parameters were as follows: Echo time $(\mathrm{TE})=17$, repetition time $(\mathrm{TR})=360$, acquisition matrix $=256 \times 100$, and slice thickness $=5 \mathrm{~mm}$. Whole-brain FLAIR images were also acquired to quantify WMH. For the FLAIR images, twenty-one 5-mm slices were acquired with $\mathrm{TR}=8500$, $\mathrm{TI}=2500$, Flip Angle $=150$ degrees, $\mathrm{TE}=115$, and $\mathrm{FOV}=220 \times 75$.

Morphometric analysis of brain structure was completed with FreeSurfer Version 5.1 (http://surfer.nmr.mgh. harvard.edu). Detailed methodology for regional and total volume derivation has been described in detail previously [28-30]. FreeSurfer was used to perform image preprocessing (e.g. intensity normalization, skull stripping), then to provide both cortical and subcortical volume measures using the surface stream and the subcortical segmentation stream respectively. FreeSurfer performs such parcellations by registering images to a probabilistic brain atlas, built from a manually labeled training set, and then using this probabilistic atlas to assign a neuroanatomical label to each voxel in an MRI volume. Total brain volume, total gray matter volume, and volume of the thalamus and brain stem were all automatically derived with the subcortical processing stream (i.e., "aseg.stats"). Intracranial volume was also automatically derived and served as a covariate in analyses examining MRI indices in order to control for interindividual differences in head size. A summary composite was computed for the left and right hemisphere volumes of the thalamus.

Total WMH volume was derived by a three-step operator-driven protocol that has been described in detail previously [31]. Briefly, in Step 1, a threshold was applied to each FLAIR image to label all voxels that fell within the intensity distribution of hyperintense signal.
In Step 2, gross regions-of-interest (ROI) were drawn manually to include WMH but to exclude other regions (e.g., dermal fat) that have similar intensity values. In Step 3, a new image is generated that contains the intersection of voxels labeled in Step 1 and those labeled in Step 2. The resulting image contains labeled voxels that are common in Step 1 and Step 2. The number of resulting voxels is summed and multiplied by voxel dimensions to derive a total volume score. The validity and reliability of this approach has been demonstrated previously [31].

\section{Cerebral blood flow}

Transcranial Doppler (TCD) ultrasonography was performed through an expanded Stroke Prevention Trial in Sickle Cell Anemia (STOP) protocol [32] and used to quantify mean cerebral blood flow velocity (CBF-V) of the Middle Cerebral Artery (MCA). The MCA was chosen to operationalize cerebral blood flow in this study for several reasons. First, it irrigates the frontal, temporal, and the parietal cerebrum and reliably reflects changes in cerebral blood flow. CBF-V of the MCA also exhibits higher blood flow velocity compared to other TCD measured arteries (e.g., ACA, PCA) [33,34]. Furthermore, persons with HF demonstrate significant reductions in CBF-V of the MCA [35] and such reductions have been linked with WMH in older adults with HF [15]. Lastly, CBF-V of the MCA was also chosen because of its known sensitivity to sleep disturbances in healthy populations [36].

\section{Estimate of HF severity/physical fitness}

The 2-minute step test (2MST) was used to assess physical fitness levels and to serve as a proxy of HF severity in the current sample [37]. The 2MST is a brief assessment that requires participants to march in place lifting his/her knees to a marked target set on the wall set at the midpoint between the kneecap and crest of the iliac for a 2-minute period. Greater step count is associated with better physical fitness and has been correlated with metabolic equivalents derived from stress testing [38]. The 2MST was included as a covariate in the current analyses to control for the effects of physical fitness on the brain in HF [39] and on sleep quality [40].

\section{Demographic and medical history}

Participants' medical and demographic history was ascertained through self-report and corroborated and supplemented by medical record review. Through these methods, information regarding participants' physician diagnostic history of diabetes, sleep apnea, hypertension, and depression as well as prescribed medications were obtained. LVEF of the current sample was obtained through medical chart review. Medical record review 
took precedence in the case of discrepancy from selfreport. Refer to Table 1.

\section{Procedures}

The Kent State University and Summa Health System Institutional Review Board (IRB) approved the study procedures and all participants provided written informed consent prior to study enrollment. At a baseline assessment, all participants completed demographic, medical history, and psychosocial self-report measures, including the PSQI. A medical record review was also completed at this baseline assessment to corroborate self-report and also ascertain LVEF of the sample; all participants completed an echocardiograph as part of their standard clinical care prior to study entry. At a separate study visit, but within 2-weeks of the baseline assessment, all participants also underwent MRI and TCD ultrasonography.

\section{Statistical methods}

All analyses were performed using SPSS software. A log transformation of WMH was performed to correct for the positively skewed distribution of this variable. A series of multivariable hierarchical regression analyses was conducted to examine the effects of adverse brain changes on sleep quality in HF. For all analyses, the global PSQI served as the continuous dependent variable. To determine the independent effects of brain indices on sleep quality, demographic and medical covariates that are known to influence neurocognitive outcomes and/or sleep quality in HF were entered into block 1 of all regression models to account for their variance. These covariates included age, LVEF, 2MST, intracranial volume, and diagnostic history of diabetes, sleep apnea, hypertension, and depression ( 1 = positive diagnostic history; $0=$ negative diagnostic history). A separate regression model was performed for each of the following continuous brain predictors that were entered in block 2, yielding six separate regression models: $\mathrm{WMH}$, total brain volume, total gray matter volume, thalamus, and brain stem volume, and CBF-V of the MCA. Intracranial volume was not controlled for in the regression model examining $\mathrm{CBF}-\mathrm{V}$ of the MCA as the predictor variable, as head size does not represent a possible confound in this analysis. Follow-up partial correlations adjusting for age, LVEF, 2MST, intracranial volume, and diagnostic history of diabetes, sleep apnea, hypertension, and depression were conducted to examine the correlations among WMH, cerebral blood flow, specific item-level sleep difficulties of the PSQI.

Lastly, bivariate correlations examined the association between CBF-V of the MCA and WMH. A regression model with the above listed covariates (but, not intracranial volume) entered in block 1 and $\mathrm{CBF}-\mathrm{V}$ of the MCA and $\mathrm{WMH}$ entered in block 2 was then performed to determine whether the combination of cerebral hypoperfusion and increased WMH independently predicted poor sleep quality.

\section{Results}

Sleep quality

Refer to Table 2 for sleep characteristics of the current sample. The sample had an average global PSQI score of $8.41(\mathrm{SD}=3.57)$ with $75.5 \%$ classified as impaired sleepers (i.e., PSQI $>5$ ). The most commonly reported sleep difficulties in the sample included interrupted sleep, nocturia, snoring or coughing fits, and pain. In terms of overall quality of sleep, $5.7 \%$ rated their quality of sleep to be very bad and $22.6 \%$ reported their sleep quality to be fairly bad.

\section{Neuroimaging, cerebral blood flow, and overall sleep quality}

See Table 3 for a full summary of demographic and medical predictors of sleep quality. After adjustment for medical and demographic variables and intracranial volume, WMH was associated with the global PSQI ( $\beta=$ $0.28, p=0.046)$. Increased WMH volume was associated with greater sleep difficulties. CBF-V of the MCA also emerged as a significant predictor of global PSQI $(\beta=-0.28, p=0.04)$, even after controlling for medical and demographic variables. See Table 4. Decreased CBF-V of the MCA was associated with poorer sleep quality. There were no significant effects on sleep quality for total brain, total gray matter volume or for any of the regional volumetric indices (i.e., thalamus or brain stem volume) $(p>.05$ for all).

\section{Sleep difficulties, WMH, cerebral blood flow}

Partial correlations adjusting for age, LVEF, 2MST, intracranial volume, and diagnostic history of diabetes, sleep apnea, hypertension, and depression showed increased WMH was associated with greater difficulty falling asleep $(r(43)=0.31, p=0.04)$, feeling too hot $(r(43)=$ $0.32, p=0.03)$, and decreased overall sleep quality $(r(43)=$ $0.39, p=.01)$. Lower CBF-V was also associated with having bad dreams $(r(43)=-0.39, p=0.01)$, decreased enthusiasm to get things done $(r(43)=-0.40, p=0.01)$, and poorer overall quality of sleep $(r(43)=-0.40, p=$ $0.01)$. Interestingly, greater $\mathrm{CBF}-\mathrm{V}$ of the MCA was associated with increased sleep medication use $(r(43)=$ $0.33, p=0.03)$.

\section{Association between WMH and cerebral blood flow}

Bivariate correlations showed that $\mathrm{CBF}-\mathrm{V}$ of the MCA demonstrated a significant association with WMH $(r(51)=-0.31, p=0.03)$. Decreased cerebral blood flow was associated with greater WMH. Regression analyses controlling for medical and demographic variables revealed that 
Table $\mathbf{2}$ Reported sleep difficulties in $\mathbf{5 3}$ older adults with heart failure

\begin{tabular}{lcc}
\hline & Once or twice per week (\%) & Three or more times per week (\%) \\
\hline Cannot Get to Sleep within 30 Minutes & 26.4 & 17.0 \\
Wake Up in the Middle of the Night or Early Morning & 30.2 & 41.5 \\
Get Up to Use the Bathroom & 26.4 & 58.5 \\
Cannot Breathe Comfortably & 7.5 & 11.3 \\
Cough or Snore Loudly & 7.5 & 26.4 \\
Feel Too cold & 1.9 & 13.2 \\
Feel Too Hot & 13.2 & 5.7 \\
Bad Dreams & 9.4 & 3.8 \\
Pain & 13.2 & 20.8 \\
Other Reasons & 11.3 & 9.4 \\
\hline
\end{tabular}

the combination of both reduced CBF-V of the MCA and increased WMH demonstrated significant predictive properties of poorer sleep quality $(F(10,42)=2.93, p=0.03)$, suggesting a possible interaction between these variables on sleep quality.

\section{Discussion}

Consistent with the extant literature, the current study shows that reported sleep difficulties are prevalent in older adults with HF. Past work has demonstrated that a series of demographic (e.g., older age) and medical factors (e.g., HF severity) are correlated with poor sleep quality in HF [5], though findings are not entirely consistent across

Table 3 WMH predict sleep quality in older adults with heart failure $(N=53)$

\begin{tabular}{ll}
\hline Variable & Global PSQI \\
& $\boldsymbol{\beta}(\boldsymbol{S E} \boldsymbol{b})$ \\
\hline Block 1 & \\
Age & $-.03(.06)$ \\
2MST & $-.36(.02)^{*}$ \\
LVEF & $-.10(.03)$ \\
Depression & $-.06(1.25)$ \\
Hypertension & $-.17(1.04)$ \\
Diabetes & $.12(1.09)$ \\
Sleep Apnea & $-.18(.00)$ \\
ICV & $-.18(.00)$ \\
$R^{2}$ & .30 \\
F & $2.41^{*}$ \\
Block 2 & \\
WMH & $.28(1.36)^{*}$ \\
$R^{2}$ & .37 \\
F for $\Delta R^{2}$ & $4.22^{*}$ \\
\hline
\end{tabular}

Note. ${ }^{*}$ denotes $\mathrm{p}<0.05$.

Abbreviations: $\beta$ standardized regression coefficients, SE standard error, 2MST 2-minute step test, LVEF left ventricular ejection fraction, ICV Intracranial volume, WMH White matter hyperintensities, PSQI Pittsburgh sleep quality index. studies [7]. The current findings extend past work by showing that adverse structural and functional brain changes may be important contributors to sleep disturbances in patients with HF.

The current study shows that greater WMH volume is associated with poorer sleep quality in older adults with HF. The exact mechanisms underlying this association are unclear, though there are several possible explanations. Disrupted circadian rhythms have been shown to occur in vascular dementia patients, believed to be the result of deafferentation $[41,42]$, and $\mathrm{WMH}$ may lead to similar circadian rhythm disturbances in HF by interfering with cortical and subcortical neuronal connections to key brain regions that help regulate sleep (e.g., brain

Table 4 Cerebral blood flow predicts sleep quality in older adults with heart failure $(N=53)$

\begin{tabular}{|c|c|}
\hline Variable & $\begin{array}{l}\text { Global PSQI } \\
\beta(S E b)\end{array}$ \\
\hline \multicolumn{2}{|l|}{ Block 1} \\
\hline Age & $.06(.06)$ \\
\hline 2MST & $.43(.02)^{*}$ \\
\hline LVEF & $-.10(.03)$ \\
\hline Depression & $-.04(1.24)$ \\
\hline Hypertension & $-.15(1.03)$ \\
\hline Diabetes & $.14(1.08)$ \\
\hline Sleep Apnea & $.21(1.12)$ \\
\hline$R^{2}$ & .28 \\
\hline$F$ & $2.53^{*}$ \\
\hline \multicolumn{2}{|l|}{ Block 2} \\
\hline CBF-V MCA & $-.28(.04)^{*}$ \\
\hline$R^{2}$ & .35 \\
\hline$F$ for $\Delta R^{2}$ & $4.41^{*}$ \\
\hline
\end{tabular}

Note. *denotes $\mathrm{p}<0.05$.

Abbreviations: $\beta$ standardized regression coefficients, SE standard error, 2MST 2-minute step test, $L V E F$ left ventricular ejection fraction, CBF-V MCA cerebral blood flow velocity of the middle cerebral artery, PSQI Pittsburgh sleep quality index. 
stem, suprachiasmatic nucleus) [43-47]. Supporting this possibility is the current association between WMH and altered core body temperature (i.e., feeling too hot as assessed by item $5 \mathrm{~g}$ on the PSQI) and past work that demonstrates $\mathrm{HF}$ patients exhibit impaired integrity of axonal projections among brain structures important for sleep, including connections among the basal forebrain, hypothalamus, raphe magnus, and brain stem [20,48]. WMH are also closely linked with amyloid beta deposition [49] and amyloid beta is a significant contributor to poor sleep quality in pre-clinical Alzheimer's disease (AD) patients [50]. This is noteworthy, as HF patients are at risk for $\mathrm{AD}$ and declining sleep quality may be indicative of $\mathrm{AD}$ pathogenesis in this population [50,51]. Lastly, the known influence of WMH on psychiatric symptoms (e.g., depression) in HF may also contribute to decreased sleep quality $[13,52]$.

Reduced cerebral blood flow also emerged as a significant predictor of sleep quality in the current sample of HF patients, and may interact with increased WMH to exacerbate sleep disturbances. Cerebral hypoperfusion and subsequent ischemia is commonly proposed to underlie WMH in HF $[15,21]$ and the current findings showed an inverse association between cerebral perfusion and WMH. Thus, it is likely that chronic disruptions in cerebral hemodynamics affects sleep in HF through its association with WMH, though the crosssectional design of the current study precludes empirical test of such mediation. Our findings are in the opposite direction of past work examining cerebral blood flow and sleep quality in AD patients [24]. Similarly, sleep spindles help to prevent arousal during sleep, and in contrast to the suggested direction of our findings, past work shows that cerebral blood flow and spindle activity are negatively correlated [25,53-56]. The exact reason for the directionality of our findings is not clear, but it is plausible that decreased cerebral blood flow in HF may reflect greater HF severity such as increased number of medical comorbidities and cardiac dysfunction, which are all significant correlates of poor sleep quality in this population [5]. Interestingly, past work shows that cerebral blood flow can be improved through physical activity interventions [57]. This is noteworthy, as improved systemic perfusion in HF has been linked with better neurocognitive outcomes [58]. Because physical activity is a key treatment recommendation in the management of $\mathrm{HF}$, future studies should examine whether greater physical activity results in better sleep quality among HF patients due to improved cerebral circulation.

The current study found no association between total and regional brain volume and sleep quality. These findings further suggest interference with cortico-cortical and cortico-subcortical connections as a likely underpinning for poor sleep quality in HF given the observed impact of WMH on reduced sleep quality in the current study. Although brain atrophy has been linked with reduced sleep quality in other medical populations [19], it is likely that atrophy in the current sample did not reach threshold to produce clinical manifestations. For instance, brain volume loss has been shown among patients with greater HF severity (i.e., LVEF <30) [20] while LVEF in this sample fell within the average range (i.e., mean LVEF $=40$ ). Longitudinal studies that examine the effects of brain atrophy on sleep in HF are needed, particularly as it involves WMH.

The current findings are limited in several ways. The cross-sectional design does not permit causal inferences and prospective studies are needed to confirm our findings. For instance, it is possible that insufficient sleep in HF may lead to reduced cerebral perfusion [23], though this is unlikely in this population in light of the negative effects of cerebral hypoperfusion on the brain [15]. In addition, self-report of sleep quality is limited by biases [59] and future work should use objective assessments of sleep quality (e.g., polysomnography). Similarly, future studies that employ imaging techniques examining axonal tractography would also help elucidate the underlying mechanisms between WMH and sleep quality through direct examination of neuronal pathways that may be occluded by the presence of WMH. We also found an inverse association between physical fitness and sleep quality. Past work shows that exercise interventions in HF lead to improved sleep quality [60] and future work should examine whether such findings are a result of the positive benefits of exercise on the brain. Lastly, although it is notable that the current study controlled for the effects of diagnosed sleep apnea, such history was obtained through self-report and medical record review and information regarding duration, treatment, and severity of sleep apnea is unknown. Consequently, it is possible that undiagnosed sleep apnea or sleep disordered breathing was present in many of the HF participants [10], which may have introduced possible confounds. Future work that employs more objective assessments of sleep quality is needed to clarify and confirm the current findings. Likewise, case controlled studies with larger samples that are more diverse in disease severity and utilize direct measurements of comorbid conditions are much needed to better understand the independent effects of brain abnormalities on sleep in HF.

\section{Conclusions}

In brief summary, the current study shows that WMH and decreased cerebral blood flow are associated with poor sleep quality in older adults with HF. Prospective studies are needed to confirm these findings, clarify mechanisms by which WMH and reduced cerebral blood 
flow disrupt sleep, and examine whether sleep difficulties are modifiable through interventions that target improved cerebral blood flow (e.g., cardiac rehabilitation).

\section{Abbreviations}

HF: Heart failure; WMH: White matter hyperintensities; LVEF: Left ventricular ejection fraction; CBF-V of the MCA: Cerebral blood flow velocity of the middle cerebral artery; 2MST: 2-minute step test.

\section{Competing interests}

The authors declare that they have no competing interests.

\section{Authors' contributions}

$M A, A M B, M B S, J G, R C$, and $L S$ participated in the design of the study, acquisition of data, and analysis and interpretation of data. MA, MBS, JG, EYG, AN, JH, JR participated in acquisition of data and analysis and interpretation of data. All authors were involved in drafting the manuscript and revising it for intellectual content. All authors also provided final approval of the version to be published.

\section{Acknowledgements}

Support for this work included National Institutes of Health $(\mathrm{NIH})$ grants DK075119 and HLO89311. The authors have no competing interests to report.

\section{Author details}

${ }^{1}$ Department of Psychology, Kent State University, Kent, OH, USA. ${ }^{2}$ Department of Neurology, Taub Institute for Research on Alzheimer's Disease and the Aging Brain, College of Physicians and Surgeons, Columbia University, New York, NY, USA. ${ }^{3}$ Department of Psychiatry, Summa Health System Akron City Hospital, Akron, OH, USA. ${ }^{4}$ Departments of Neurology Psychiatry and the Institute on Aging, Center for Cognitive Aging and Memory, University of Florida, Florida, Gainesville, USA. ${ }^{5}$ Department of Psychology, University of Georgia, Athens, USA.

Received: 21 August 2013 Accepted: 27 October 2013

Published: 30 October 2013

\section{References}

1. Pressler SJ, Subramanian U, Kareken D, Perkins SM, Gradus-Pizlo I, Suave MJ, Ding Y, Kim J, Sloan R, Jaynes H, Shaw RM: Cognitive deficits in chronic heart failure. Nurs Res 2010, 59:127-139.

2. Go AS, Mozaffarian D, Roger VL: Heart disease and stroke statistics-2013 update. Circulation 2013, 127:e6-e245.

3. Bennett SJ, Oldridge NB, Eckert G, Embree JL, Browning S, Hou N, Hou N Chui M, Deer M, Murray MD: Comparison of quality of life measures in heart failure. Nurs Res 2003, 52:207-216.

4. Tremel F, Pepin JL, Veale D, et al: High prevalence and persistence of sleep apnoea in patients referred for acute left ventricular failure and medically treated over 2 months. Eur Heart J 1999, 20:1201-1209.

5. Wang TJ, Lee SC, Tsay SL, et al: Factors influencing heart failure patients sleep quality. J Adv Nurs 2010, 66:1730-1740.

6. Dos Santos MA, Guedes Ede S, Barbosa RL, Da Cruz Dde A: Sleeping difficulies reported by patients with heart failure. Rev Lat Am Enfermagem 2012, 20:644-650.

7. Erickson VS, Westlake CA, Dracup KA, Woo MA, Hage A: Sleep disturbance symptoms in patients with heart failure. AACN Clin Issures 2003, 14:477-487.

8. Redeker NS: Sleep disturbance in people with heart failure: implications for self-care. J Cardiovasc Nurs 2008, 23:231-238.

9. Redeker NS, Stein S: Characteristics of sleep in patients with stable heart failure versus a comparison group. Heart Lung 2006, 35:252-261.

10. Redeker NS, Muench U, Zucker MJ, et al: Sleep disordered breathing, daytime symptoms, and functional performance in stable heart failure. Sleep 2010, 33:551-560.

11. Garcia S, Alosco ML, Spitznagel MB, et al: Poor sleep quality and reduced cognitive function in persons with heart failure. Int $J$ Cardiol 2012, 156:248-249.

12. Izawa KP, Watanabe S, Oka K, Hiraki K, et al: Relation between sleep quality and physical activity in chronic heart failure patients. Recent Pat Cardiovasc Drug Discov 2011, 6:161-167.

13. Parker KP, Dunbar SB: Sleep and heart failure. J Cardiovasc Nurs 2002, 17:30-41.
14. Foley DJ, Monjan A, Simonsick EM, et al: Incidence and remission of insomnia among elderly adults:an epidemiologic study of 6,800 persons over three years. Sleep 1999, 22:S366-S372.

15. Alosco ML, Brickman AM, Spitznagel MB, et al: Cerebral perfusion is associated with white matter hyperintensities in older adults with heart failure. Congest Heart Fail 2013. epub ahead of print.

16. Beer C, Ebenezer E, Fenner S, et al: Contributor to cognitive impairment in congestive heart failure: a pilot case-control study. Intern Med J 2009, 39:600-605

17. Vogels RL, van der Flier WM, van Harten $B$, et al: Brain magnetic resonance imaging abnormalities in patients with heart failure. Eur J Heart Fail 2007, 9:1003-1009.

18. Woo MA, Kumar R, Macey PM, et al: Brain injury in autonomic, emotional, and cognitive regulatory areas in patients with heart failure. $J$ Card Fail 2009, 15:214-223.

19. Gama RL, Tavora DG, Bomfirm RC, Silva CE, De Bruin VM, De Bruin PF: Sleep disturbances and brain MRI morphometry in Parkinsons disease, multiple system atrophy and progressive supranuclear palsy-a comparative study. Parkinsonism Relat Disord 2010, 16:275-279.

20. Kumar R, Woo MA, Macey PM, et al: Brain axonal and myelin evaluation in heart failure. J Neurol Sci 2011, 307:106-113.

21. Gruhn N, Larsen FS, Boesgaard S, Knudsen GM, Mortensen SA, Thomsen G, Aldershville J: Cerebral blood flow in patients with chronic heart failure before and after heart transplantation. Stroke 2001, 32:2530-2533.

22. Celle S, Peyron R, Faillenot I, et al: Undiagnosed sleep-related breathing disorders are associated with focal brainstem atrophy in the elderly. Hum Brain Mapp 2009, 30:2090-2097.

23. Miyata S, Noda A, Ozaki N, et al: Insufficient sleep impairs driving performance and cognitive function. Neurosci Lett 2010, 469:229-233.

24. Ismail Z, Herrmann N, Francis PL, et al: A SPECT study of sleep disturbance and Alzheimer's disease. Dement Geriatr Cogn Disord 2009, 27:254-259.

25. Hofle N, Paus T, Reutens D, Fiset P, et al: Regional cerebral blood flow changes as a function of delta and spindle activity during slow wave sleep in humans. J Neurosci 1997, 17:4800-4808.

26. Gvilia I: Underlying brain mechanisms that regulate sleep-wakefulness cycles. Int Rev Neurbiol 2010, 93:1-21.

27. Buysse DJ, Reynolds CF III, Monk TH, et al: The Pittsburgh Sleep Quality Index: A new instrument for psychiatric practice and research. Psychiatry Res 1989, 28:193-213.

28. Fischl B, Salat DH, Busa E, Albert M, Dieterich M, Haselgrove C, et al: Whole brain segmentation: automated labeling of neuroanatomical structures in the human brain. Neuron 2002, 33:341-355.

29. Fischl B, Sereno MI, Dale AM: Cortical surface-based analysis. II: Inflation, flattening, and a surface-based coordinate system. Neuroimage 1999, 9:195-207.

30. Fischl B, van der Kouwe A, Destrieux C, Halgren E, Segonne F, Salat DH, et al: Automatically parcellating the human cerebral cortex. Cereb Cortex 2004, 14:11-22.

31. Brickman AM, Sneed JR, Provenzano FA, Garcon E, Johnert L, Muraskin J, et al: Quantitative approaches for assessment of white matter hyperintensities in elderly populations. Psychiatry Res 2011, 193:101-106.

32. Bulas D, Jones A, Seibert J, Driscoll C, O'Donnell R, Adams RJ: Transcranial Doppler (TCD) screening for stroke prevention I sickle cell anemia: pitfalls in technique variation. Pediatr Radiol 2000, 30:733-738.

33. Bishop CC, Powell S, Rutt D, Browse NL: Transcranial Doppler measurement of middle cerebral artery blood flow velocity: a validation study. Stroke 1986, 17:913-915.

34. Macchi $C$, Catini $C$ : The measuremnt of the calibers and blood-flow velocities of the arterieis of the circle of willis: a statistical investigation of 120 living subjects using transcrnial colo-Doppler ultrasonogrpahy. Ital J Anat Embryol 1994, 99:9-16.

35. Vogels RL, Oosterman JM, Laman DM, et al: Transcrandial Doppler blood flow assessment in patients with mild heart failure: correlates with neuroimaging and cognitive performance. Congest Heart Fail 2008, 14:61-65.

36. Netzer $N$, Werner $P$, Jochums I, et al: Blood flow of the middle cerebral artery with sleep-disordered breathing: Correlation with obstructive hypneas. Stroke 1998, 29:87-93.

37. Jones CJ, Rikli RE: Measuring functional fitness of older adults. The Journal on Active Aging 2002:24-30 
38. Garcia S, Alosco ML, Spitznagel MB, et al: Cardiovascular fitness associated with cognitive performance in heart failure patients enrolled in cardiac rehabilitation. BMC Cardiovasc Disord 2013, 13:29.

39. Alosco ML, Brickman AM, Spitznagel MB, et al: Poorer physical fitness is associated with reduced structural brain integrity in heart failure. J Neurol Sci 2013. epub ahead of print.

40. Loprinzi PD, Cardinal BJ: Association between objectively-easured physical activity and sleep, NHANES 2005-2006. Mental Health and Physical Activity 2011, 4:65-69.

41. Aharon-Peretz J, Masiah A, Pillar T, Epstein R, Tzischinsky O, Lavie P: Sleepwake cycles in multi-infarct dementia and dementia of the Alzheimer type. Neurology 1991, 41:1616-1619.

42. Mishima K, Okawa M, Satoh K, Shimizu T, Hozumi S, Hishikawa Y: Different manifestations of circadian rhythms in senile dementia of Alzheimer's type and multi-infarct dementia. Neurobiol Aging 1997, 18:105-109.

43. Oosterman J, van Harten B, Vogels R, Gouw A, Weinstein H, Scheltens P, Scherder $\mathrm{E}$ : Distortions in rest-activity rhythm in aging relate to white matter hyperintensities. Neurobiol Aging 2008, 29:1265-1271.

44. Kanda A, Matsui T, Ebihara S, Arai H, Sasaki H: Periventricular white matter lesions and sleep alteration in older people. J Am Geriatr Soc 2003, 51:432-433.

45. Meguro K, Ueda M, Kobayashi I, Yamaguchi S, Yamazaki H, Oikawa Y, Kikuchi $Y$, Sasaki H: Sleep disturbance in elderly patients with cognitive impairment, decreased daily activity and periventricular white matter lesions. Sleep 1995, 18:109-114.

46. Moore RY, Speh JC, Lezak RK: Suprachiasmatic nucleus organization. Cell Tissue Res 2002, 309:89-98.

47. Pace-Schott EF, Hobson JA: The neurobiology of sleep: genetics, cellular physiology and subcortical networks. Nat Rev Neurosci 2002, 3:591-605.

48. Weldemichael DA, Grossberg GT: Circadian rhythm-distrubances in patients with Alzheimer's disease: A review. Int J Alzeimers Dis 2010, 2:716453.

49. Gurol ME, Irizarry MC, Smith EE, et al: Plasma beta-amyloid and white matter lesions in $\mathrm{AD}, \mathrm{MCl}$, and cerebral amyloid angiopathy. Neurology 2006, 66:23-29.

50. Ju YS, McLeland JS, Toedebusch CD, et al: Sleep quality and preclinical Alzheimer disease. JAMA Neurol 2013, 11:1-7.

51. Qiu C, Winblad B, Marengoni A, et al: Heart failure and risk of dementia and Alzheimer disease: a population-based cohort study. Arch Intern Med 2006, 166:1003-1008.

52. Almeida JR, Alves TC, Wajngarten M, et al: Late-life depression, heart failure and frontal white matter hyperintensities : a structural magnetic resonance imaging study. Braz J Med Biol Res 2005, 38:431-436.

53. Coenen AM: Neuronal activities underlying the electroencephalogram and evoked potentials of sleeping and waking: implications for information processing. Neurosci Biobehav Rev 1995, 19:447-463.

54. Elton M, Winter O, Heslenfeld D, Loewy D, Campbell K, Kok A: Event-related potentials to tones in the absence and presence of sleep spindles. J Sleep Res 1997, 6:78-83.

55. Lopes Da Silva F: Neural mechanisms underlying brain waves: from neural membranes to networks. Electroencaphalogr Clin Neurophysiol 1991, 79:81-93.

56. Steriade M, Amzica F: Coalescence of sleep rhytms and their chronology in corticothalamic networks. Sleep Res Online 1998, 1:1-10.

57. Bailey DM, Marley CJ, Brugniaux JV, et al: Elevated aerobic fitness sustained throughout the adult lifespan is associated with improved cerebral hemodynamics. Stroke 2013. epub ahead of print.

58. Bornstein RA, Starling RC, Myerowitz PD, et al: Neuropsychological function in patients with end-stage heart failure before and after cardiac transplantation. Acta Neurol Scand 1995, 91:260-265.

59. Perils ML, Giles DE, Mendelson WB, Bootzin RR, Wyatt JK: Psychophysiological insomnia. The behavioural model and a neurocognitive perspective. J Sleep Res 1997, 6:179-188.

60. Gary R, Lee SY: Physical function and quality of life in older women with diastolic heart failure: effects of a progressive walking program on sleep patterns. Prog Cardiovasc Nurs 2007, 22:72-80.

doi:10.1186/1744-9081-9-42

Cite this article as: Alosco et al:: Reduced cerebral blood flow and white matter hyperintensities predict poor sleep in heart failure. Behavioral and Brain Functions 2013 9:42.

\section{Submit your next manuscript to BioMed Central and take full advantage of:}

- Convenient online submission

- Thorough peer review

- No space constraints or color figure charges

- Immediate publication on acceptance

- Inclusion in PubMed, CAS, Scopus and Google Scholar

- Research which is freely available for redistribution 